\title{
ON MEAN VALUES OF FOURIER TRANSFORMS
}

\author{
Michel J. G. WEBER
}

Abstract. We show that there exists a sequence $\left\{n_{k}, k \geqslant 1\right\}$ growing at least geometrically such that for any finite non-negative measure $v$ such that $\widehat{v} \geqslant 0$, any $T>0$,

$$
\int_{-2^{n_{k}} T}^{2^{n_{k}} T} \widehat{v}(x) \mathrm{d} x \ll_{\varepsilon} T 2^{2^{(1+\varepsilon) n_{k}}} \int_{\mathbb{R}}\left|\frac{\sin x T}{x T}\right|^{n_{k}^{2}} v(\mathrm{~d} x) .
$$

Mathematics subject classification (2010): Primary 60F15, 60G50; secondary $60 \mathrm{~F} 05$.

Keywords and phrases: Fourier transform, mean value, convolution products, dilation, shift.

\section{REFERENCES}

[1] D. S. Mitrinović, Analytic inequalities, Springer Verlag 165, (1970).

[2] M. WEBER, Dirichlet polynomials: some old and recent results, and their interplay in number theory, Dependence in probability, analysis and number theory, 323-353, (2010), Kendrick Press, Heber City, UT.

[3] M. Weber, On Mean Values of Dirichlet Polynomials, Math. Inequalities \& Appl. 14 (3), (2011), $529-534$ 